\title{
Technè
}

La science au service de l'histoire de l'art et de la préservation des biens culturels

44 | 2016

Archives de l'humanité : les restes humains patrimonialisés

\section{Imagerie médicale des restes humains anciens}

Medical imaging of ancient human remains

\section{Samuel Mérigeaud}

\section{(2) OpenEdition}

1 Journals

\section{Édition électronique}

URL : http://journals.openedition.org/techne/1010

DOI : 10.4000/techne.1010

ISSN : 2534-5168

Éditeur

C2RMF

\section{Édition imprimée}

Date de publication : 1 novembre 2016

Pagination : 50-54

ISBN : 978-2-7118-6339-6

ISSN : 1254-7867

\section{Référence électronique}

Samuel Mérigeaud, «Imagerie médicale des restes humains anciens », Technè [En ligne], 44 | 2016, mis en ligne le 19 décembre 2019, consulté le 23 juillet 2020. URL : http://journals.openedition.org/ techne/1010 ; DOI : https://doi.org/10.4000/techne.1010

\section{c) (i) (9)}

La revue Technè. La science au service de l'histoire de l'art et de la préservation des biens culturels est mise à disposition selon les termes de la Licence Creative Commons Attribution - Pas d'Utilisation Commerciale - Pas de Modification 4.0 International. 


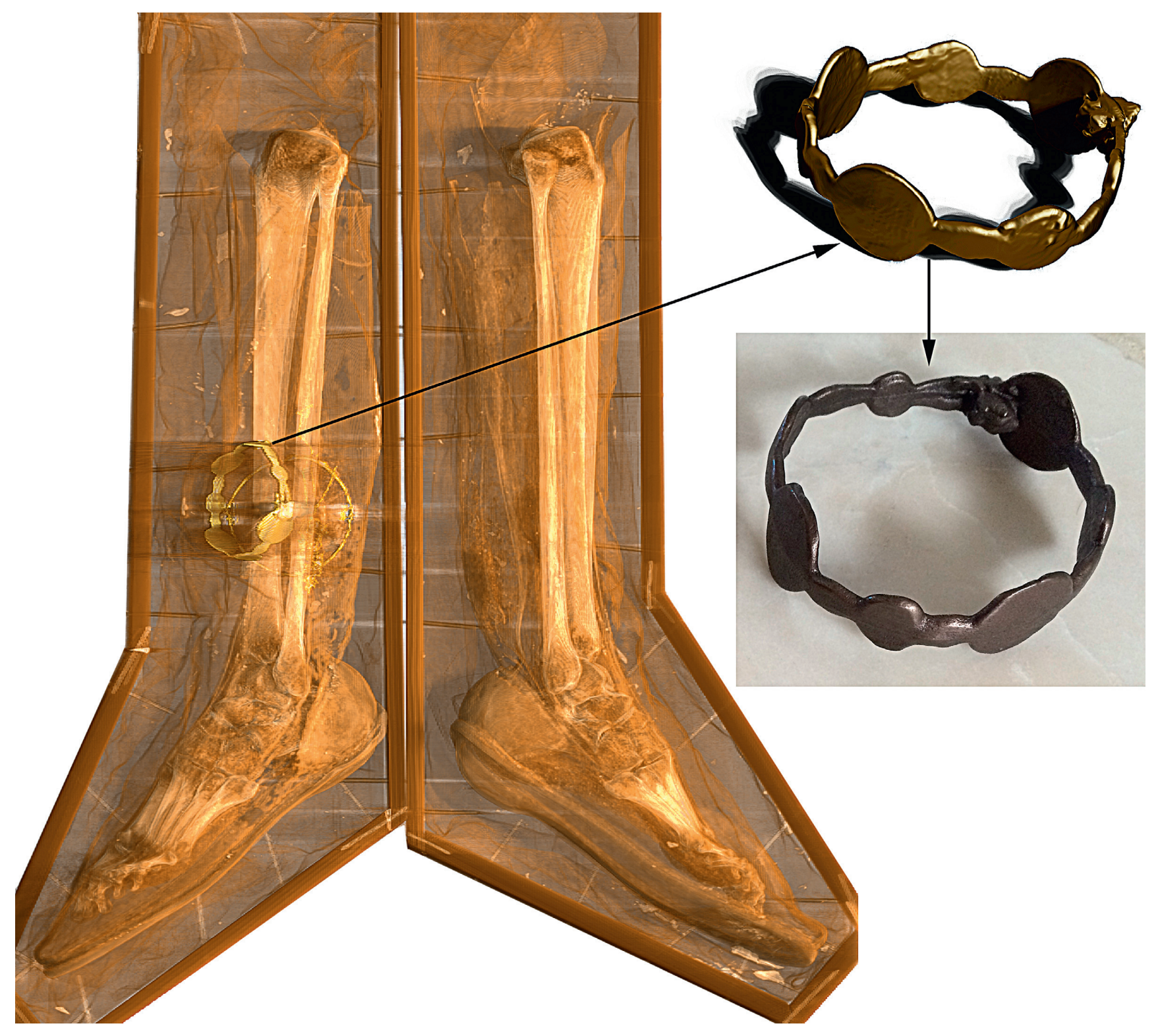

Fig. 1. Images et impression 3D de bracelets métalliques trouvés dans une botte de momie copte. @ $\odot$ S. Mérigeaud/Tridilogy/Musée du Louvre/SRDAI/Musée d'Anatomie de la faculté de médecine, Montpellier. 
Samuel Mérigeaud

\title{
Imagerie médicale des restes humains anciens
}

\author{
Medical imaging of ancient human remains
}

Résumé. Limagerie médicale est un moyen important d'étude des restes humains anciens, reposant en grande partie sur la radiographie et le scanner. Si leur utilisation se démocratise, ces techniques nécessitent cependant des connaissances spécifiques pour être efficaces, toujours dans un cadre multidisciplinaire.

Mots-clés. Scanner, tomodensitométrie, computed tomography, radiologie, radiographie, IRM, microscanner, micro CT, momie, squelette, restes humains anciens, imagerie 3D, impression $3 D$.
Abstract. Depending largely on X-rays and computed tomography, medical imaging is an important means of studying ancient human remains. While their use is becoming more widespread, these techniques nevertheless require specific knowledge in order to be efficient, always within a multidisciplinary framework.

Keywords. Scanner, CT (computed tomography) scans, radiology, X-rays, MRI, microscanner, micro CT, mummy, skeleton, ancient human remains, 3D imagery, 3D printing.
Dans l'appellation « restes humains patrimonialisés », le mot central est « humains ». Comment étudier au mieux les êtres humains d'hier sinon avec les moyens de l'imagerie médicale? L'histoire de la radiologie des momies est aussi ancienne que celle de l'imagerie médicale : inventée en 1895, la radiographie est utilisée un an après sur des momies. Au Xx $\mathrm{X}^{\mathrm{e}}$ siècle, la radiographie reste l'examen de référence, laissant petit à petit sa place au scanner au début du XXI ${ }^{\mathrm{e}}$ siècle, les autres techniques d'imagerie (microscanner, IRM) étant moins utilisées.

\section{Techniques d'imagerie pour l'étude des restes humains anciens}

La radiographie est devenue classique dans l'étude des momies. Des rayons X traversent plus ou moins un objet selon sa composition : l'eau et l'air les absorbent peu, alors que le calcium (os) et les métaux les absorbent beaucoup. Un détecteur (autrefois «photographique », aujourd'hui numérique), placé de l'autre côté de l'objet, permet de créer une image. La radiographie est donc une imagerie de projection : l'objet en entier est projeté sur une seule image plane, rendant difficile la localisation des différents constituants (on ne sait ce qui est devant ou derrière, par exemple). Il est alors nécessaire de multiplier les incidences pour bien comprendre la structure d'un objet, ce qui n'est pas toujours possible. Son grand intérêt est d'être réalisable sur site (musée ou fouille) grâce aux systèmes mobiles. Cela permet d'appliquer cette imagerie à de larges populations qui ne seraient pas accessibles autrement ${ }^{1}$.

Le scanner ou tomodensitométrie (TDM ou CT pour Computed Tomography) a révolutionné l'imagerie médicale et s'est emparé de l'imagerie des restes humains anciens ${ }^{2}$ $\left(\right.$ mais aussi animaux $^{3}$ ) depuis une dizaine d'années. Le sujet est placé sur une table adaptée au corps humain et soumis aux rayons X. À la différence de la radiographie, on n'obtient pas une seule image de projection, mais des centaines ou des milliers d'images : le sujet est « découpé » virtuellement en fines coupes (infra millimétriques). Ces images natives faites dans un plan sont ensuite intégrées dans un logiciel de lecture qui recalcule des images dans tous les plans de l'espace. Ainsi, il est plus facile de se focaliser sur une zone d'intérêt pour faire une description précise (fig. 2). Une fois l'interprétation faite sur ces images 2D, des reconstructions en 3D peuvent être réalisées pour communiquer avec les autres membres de l'équipe ou le public. Il est même possible d'isoler certains constituants (segmentation) pour mieux les visualiser en 3D (fig. 3) ou même les imprimer en 3D.

Les appareils sont de plus en plus accessibles et rapides (30 minutes d'examen). Les seules contraintes sont de déplacer la momie jusqu'au centre de radiologie et de faire attention à ne pas diffuser d'éventuelles spores dans la salle de scanner (afin de préserver les patients suivants qui seraient susceptibles d'être immunodéprimés).

Le microscanner (micro CT), dérivé du scanner médical classique, utilise également des rayons $\mathrm{X}$, mais avec une résolution plus grande et donc davantage de détails. En revanche,

Samuel Mérigeaud, Docteur en Médecine (MD), spécialiste en imagerie médicale, CRP, Clinique du Parc, Castelnau-Le-Lez, France, directeur de Tridilogy (samuelmerigeaud@yahoo.fr). 


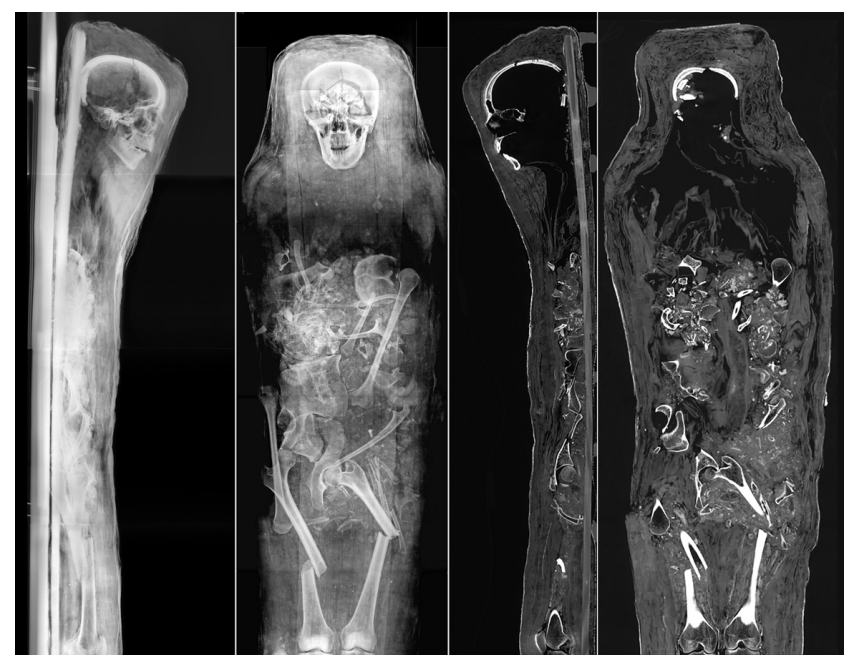

Fig. 2. Radiographies (les 2 images à gauche) et scanner (les 2 images à droite) d'une momie. La radio donne la structure générale, mais les os se superposent. Sur le scanner, chaque structure est détaillée précisément. () Radiographie C2RMF/T. Borel.

(C) Scanner S. Mérigeaud/Musée du Louvre/SRDAI/CHU Rennes/ Musée des Beaux-Arts de Rennes.

la taille de la zone étudiée est souvent réduite à quelques centimètres, voire quelques dizaines de centimètres, ce qui limite son utilisation à de petits objets. Enfin, le nombre de coupes produites est très important, nécessitant des équipements informatiques puissants pour les traiter.

Le rôle de l'IRM dans l'étude des restes humains anciens est anecdotique car elle est fondée sur l'étude de l'hydrogène, atome surtout présent dans l'eau. Les momies ou squelettes étant déshydratés, certains chercheurs ont réussi à obtenir des images mais demeurant de mauvaise qualité. Par ailleurs, certaines momies intègrent des objets métalliques pouvant se déplacer du fait du champ magnétique et abîmer la momie, voire l'IRM. Enfin, on ne peut être certain de l'absence d'effets indésirables sur la momie, tels des échauffements ou des vibrations.

\section{Comment choisir l'imagerie la plus adaptée?}

Comme nous venons de le voir, l'IRM n'a pas d'intérêt prouvé pour l'instant. Le micro CT est très intéressant pour étudier une petite zone sur un fragment de momie humaine ou sur une momie animale, mais il n'est actuellement pas envisageable de pratiquer une imagerie de ce type sur une momie humaine complète.

De nos jours, le choix se fait donc entre la radiologie et le scanner. S'il n'y a pas de risque à la mobilisation de la momie, le scanner va être privilégié car sa puissance diagnostique est bien plus importante. En revanche, la radiographie reste tout à fait d'actualité pour l'étude sur site des momies fragiles non déplaçables en dehors des musées ou bien lors de fouilles.

\section{Pièges de l'imagerie}

L'imagerie médicale produit des images qui sont une représentation de la réalité de la momie. En radiographie comme au scanner, l'appareil nécessite des réglages spécifiques, différents de ceux utilisés chez le sujet vivant. Le problème, lié en particulier au scanner, est le format particulier (DICOM) qui ne peut être lu que par des logiciels spécialisés. Ces images peuvent être si nombreuses (jusqu'à 5000 images par série et jusqu'à 5 Go de données par momie) que même les logiciels médicaux classiques ont parfois du mal à les ouvrir.

Une fois résolu ce problème de visualisation vient l'interprétation des images. Il est très important de souligner qu'au scanner, il convient de lire d'abord les images 2D dans les différents plans de l'espace, sans se précipiter sur la 3D : c'est malheureusement une erreur répandue, dommageable pour plusieurs raisons. Tout d'abord, les images natives contiennent plus d'informations grâce à leurs résolutions spatiales et, en contraste, bien plus fines que sur les images 3D. Par ailleurs, il y a des « artefacts », terme d'imagerie différent de celui utilisé en archéologie, désignant des anomalies dans l'image, liées à la technique. Ainsi, le métal diffracte les rayons X et crée de fausses images linéaires. Autre exemple, les parties très fines de certains os de la face peuvent disparaître en 3D alors qu'ils sont bien présents, causant alors des erreurs d'interprétation.

Une fois que l'interprétation est bien avancée en 2D, le passage à l'analyse en 3D permet de mieux comprendre certains éléments comme par exemple les relations entre les objets et leur disposition dans l'espace. L'imagerie 3D est également très utile pour l'analyse anthropologique, en particulier pour la détermination du sexe d'après la morphologie osseuse, mais aussi en paléopathologie, pour cartographier et analyser les fractures. Certains organes, os ou objets d'intérêt (amulettes, bandelettes...), peuvent être segmentés en 3D (fig. 1), c'est-à-dire isolés, pour mieux les représenter ou les imprimer en $3 \mathrm{D}$ : ils peuvent ainsi être manipulés directement à l'échelle. Ces outils sont très intéressants pour communiquer au sein de l'équipe ou bien avec le public ${ }^{4}$, mais ils ne sont que la dernière étape du processus et ne doivent pas remplacer l'analyse première des images 2D.

\section{Conclusion}

Après plus d'un siècle de suprématie de la radiographie, le scanner s'impose progressivement dans l'étude des restes humains anciens. Cela ne fait que suivre logiquement ce qui s'est passé en médecine humaine puis vétérinaire. On peut s'en féliciter étant donné la quantité d'informations que l'on est à même de recueillir ainsi. Mais il faut cependant être prudent car si le scanner est plus accessible, il n'en reste pas moins un examen très spécialisé en termes de réalisation et surtout d'interprétation. Il est dommage de voir certains examens réalisés uniquement pour faire des présentations $3 \mathrm{D}$ au public, avec une analyse "médicale » très succincte, 
voire absente, probablement parce que peu de personnes sont formées à cet exercice. Même les médecins radiologues sont en difficulté et font de grossières erreurs sur des scanners de momies car ils n'ont pas l'habitude des remaniements post mortem des organes momifiés observés au scanner. C'est pourquoi ce type d'étude en imagerie doit toujours être intégré dans un cadre multidisciplinaire : les échanges entre spécialités diminuent les erreurs et enrichissent les interprétations.

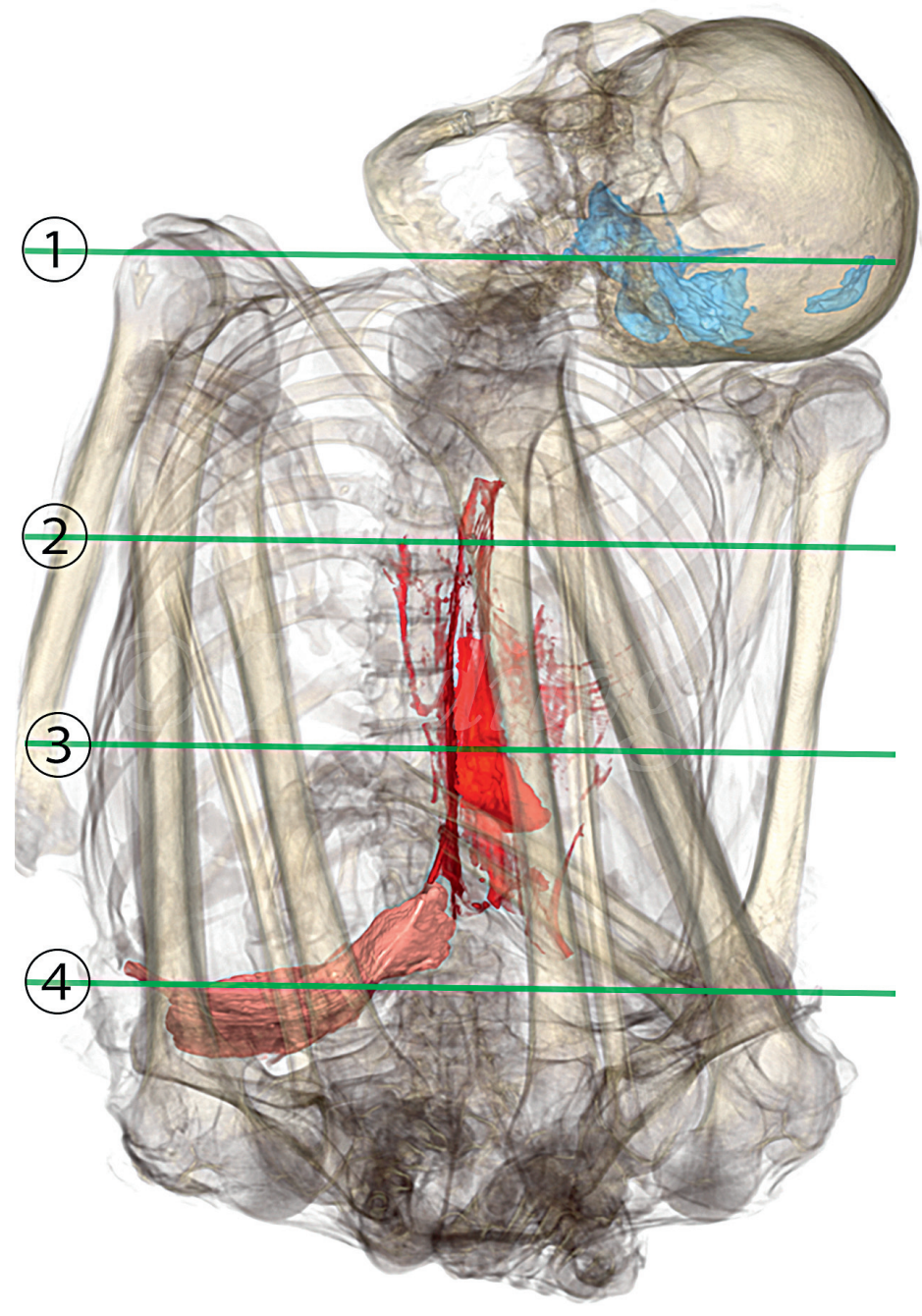

Fig. 3. Organes d'une momie bolivienne.

À gauche, image 3D avec des lignes localisant les coupes 2D.

1. Cerveau (bleu) 2. Calcification (séquelle de tuberculose ?)

3. Cour (rouge) 4 . Foie (rose).

(c) S. Mérigeaud/Tridilogy/Centre Hospitalier Geneviève-de-Gaulle Anthonioz de Saint-Dizier/Musée de Saint-Dizier.

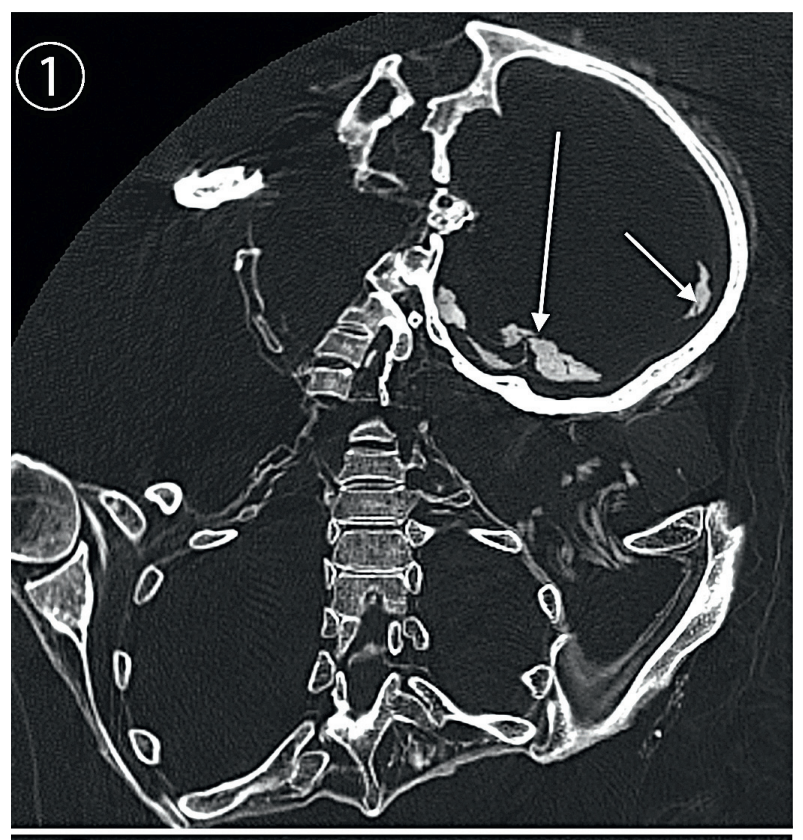

53
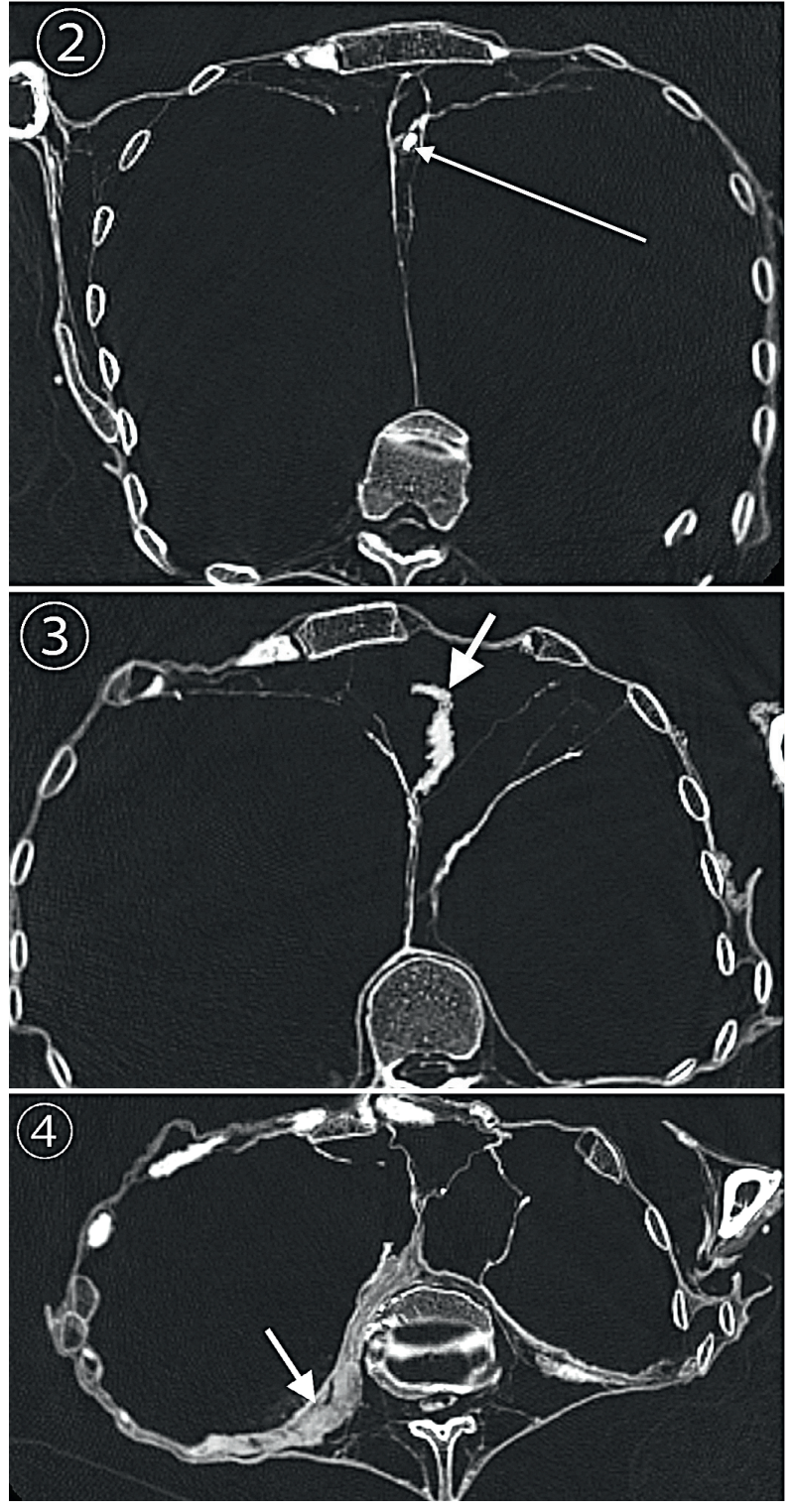


\section{Notes}

1. Exemple du travail du Dr Roger Lichtenberg avec Françoise Dunand. Il a radiographié des centaines de momies dans les fouilles des oasis de l'Ouest égyptien (El-Deir près de Kharga) et amélioré les connaissances sur l'état de santé de cette population. Dunand, 2010, 77. Lichtenberg, 2001, p. 75-77.

2. Mérigeaud, 2013, p. 61

3. Mérigeaud, 2014, p. 278.

4. Des résultats de scanners furent un élément central pour une exposition au British Museum en 2014. Exp. Londres, 2014.

\section{Bibliographie}

Dunand F, Heim J.-L., Lichtenberg R., 2010, El-Deir Nécropoles. I: La nécropole Sud, éditions Cybèle, Paris, p. 77, 87, 128 et 145 .

Exp. Londres, 2014 : Ancient lives, new discoveries [Exposition. Londres, The British Museum, 22 May-30 Novembre 2014].

Lichtenberg R., 2001, "Plusieurs cas de tuberculose diagnostiqués par la radiographie des momies de la nécropole d'Aïn-Labakha, oasis de Kharga (Égypte) ", Anthropologie, 39, 1, p. 75-77.
Mérigeaud S., 2013, «Imagerie médicale appliquée aux momies ", dans Y. Lintz et M. Coudert (dir.), Antinoé. Momies, textiles, céramiques et autres antiques, Histoire des collections du Musée du Louvre, Somogy Éditions d'art, Louvre éditions, Paris, p. 61-65 et p. 177-253.

Mérigeaud S., 2014, «La tomodensitométrie des momies animales ", dans $\mathrm{H}$.

Guichard (dir.), Des Animaux et des Pharaons. Le règne animal dans l'Egypte ancienne, Somogy Éditions d'art, Musée du Louvre-Lens, LaCaixa Foundation, Paris, p. 278-279. 